\title{
O desenvolvimento de capacidades e recursos em incubadoras de empresas
}

\section{The capability development and resources in business incubators}

\author{
José Eduardo Storopoli ${ }^{1}$ \\ Emerson AntonioMaccari² \\ Cibele Barsalini Martins ${ }^{3}$ \\ Marcelo Pereira Binder ${ }^{4}$
}

\begin{abstract}
Resumo
As incubadoras permitem que as capacidades e recursos disponíveis deem suporte ao desenvolvimento das empresas incubadas. Este trabalho tem como objetivo analisar, sob a ótica da Visão Baseada em Recursos (RBV), como as incubadoras contribuem para o desenvolvimento de suas empresas incubadas durante o processo de incubação. Foi elaborado um estudo de casos múltiploscom empresas incubadas e foram analisados os dados pelas técnicas de agrupamento e de cluster. Para as empresas em estágio inicial de incubação, a infraestrutura tem uma importância maior em relação aquelas em estágio mais avançado de incubação, porém o networking foi apontado como um dos principais benefícios proporcionado pela incubadora.
\end{abstract}

Palavras-chave: Incubadoras. Capacidades. Visão baseada em recursos.

Doutorando em Administração, Mestre em Administração pela Universidade Nove de Julho (UNINOVE) e professor da UNINOVE. E-mail: storopoli@me.com

2 Doutor em Administração pela Universidade de São Paulo - USP com Estágio Doutoral na Universityof Massachusetts Amherst - USA (2007). Mestre em Administração pela Universidade Regional de Blumenau - FURB (2002). Graduado em Administração (2000) e em Ciências da Computação (1996) pela FURB. Pesquisador Bolsista de Produtividade (PQ2/CNPq). E-mail: maccari@uninove.br

3 Professora na Universidade Federal de Santa Catarina UFSC. Doutora (2013) e Mestre (2004) em Administração pelo PPGA da Universidade Nove de Julho. É editora científica da Revista Gestão e Secretariado e presidente da Associação Brasileira de Pesquisa em Secretariado (ABPSEC). E-mail: cibelebm@uol.com.br

4 Graduado, mestre e doutor em Administração de Empresas pela Fundação Getúlio Vargas - SP (1996). Especialização pela Harvard University. Desde 2003 é professor da Fundação Getúlio Vargas - SP. E-mail: marcelo.binder@fgv.br 


\section{Abstract}

Business incubators allow that available resources and capabilities support the development of its business tenants. This study aims to analyze, through the Resource-Based View - RBV theory, how incubators contribute to the development of its tenants during the incubation process. We prepared a case study analysis with multiple incubated companies and data were analyzed by grouping and clusters techniques. For companies in incubation's early stages, the infrastructure is more important than the ones in advanced stages. However, the networking available by the incubator emerged as one of the main advantages of the incubator.

Keywords: Incubators. Capabilities. Resourced-Based View.

\section{Introdução}

A primeira iniciativa de incubação no Brasil ocorreu em 1976 (BIAGIO, 2006). Para Hannon (2003), Gassman e Becker (2006), Souza, Sousa e Bonilha (2008), Raupp e Beren (2009), Sun e Leung (2007), com a possibilidade de ter à disposição infraestruturas adequadas, desenvolver os recursos necessários e acessar as capacidades relevantes para o sucesso dos negócios incubados, os empreendedores interessados em desenvolver e lançar novos produtos notaram que as incubadoras constituíam uma maneira viável para criar e sustentar as novas empresas. De acordo com McAdam e Marlow (2007), a incubadora de negócios procura vincular efetivamente talento, tecnologia, capital e know-how para alavancar e acelerar o desenvolvimento de novas empresas.

Raupp e Beuren (2009) argumentaram que incubadoras procuram promover a redução da taxa de mortalidade de micro e pequenas empresas ajudando-as em uma melhor estruturação por meio do suporte administrativo, financeiro e de estrutura, que é disponibilizado às empresas durante o processo de incubação. De acordo com o SEBRAE (2011), a taxa de mortalidade de micro e pequenas empresas brasileiras com até dois anos de existência era de $22 \%$, sendo que os principais fatores foram: falhas gerenciais; causas econômicas conjunturais; políticas públicas e arcabouço legal; e carga tributária elevada. Portanto, 
as incubadoras promovem as novas empresas, auxiliando-as em sua sobrevivência e crescimento.

O processo de incubação é a maneira na qual as empresas incubadas se relacionam com sua incubadora na troca de informações e recursos. Tal processo não tem uma duração definida, mas é composto por diferentes estágios de amadurecimento da empresa incubada e de seus produtos até que se atinja o ponto no qual a incubada está pronta para ser lançada de maneira plena ao mercado. De certa maneira, as incubadoras formam capacidades (Hannon, 2003) em suas incubadas por meio da oferta de recursos e serviços (SUN; LEUNG, 2007; SOUZA; SOUSA; BONILHA, 2008; RAUPP; BEREN, 2009; XU, 2010). Sendo assim, torna-se importante que os estudos relacionados à contribuição das incubadoras para o desenvolvimento das empresas incubadas, sob o escopo das capacidades e recursos levem em consideração o processo de incubação em seus diferentes estágios.

A teoria da visão baseada em recursos, ou resource-based view (RBV), conhecida também como teoria dos recursos, é uma abordagem que enfatiza a categorização e análise de uma empresa como proprietária de recursos que podem gerar vantagem competitiva. Tomando-se por base a análise por recursos, é possível encontrar a otimização das atividades produto-mercado. Dentro da RBV, ao longo do tempo, desenvolveu-se uma vertente que examina as capacidades de uma empresa, em especial sua formação e consolidação (NELSON; WINTER, 1982; COLLIS, 1994; ZAHRA; SAPIENZA; DAVIDSSON, 2006). Existem diversos pontos de discussão, sob a luz da teoria da RBV, a respeito da natureza das capacidades desenvolvidas por empresas (TEECE; PISANO; SHUEN 1997; AUTIO; GEORGE; ALEXY, 2011).

Nessa linha, Baêta, Borges e Tremblay (2006) afirmaram que as incubadoras de empresas aliam as capacidades e recursos para o desenvolvimento do incubado. Neste sentido, a literatura aponta que as capacidades e recursos são desenvolvidos na incubadora pelo processo de incubação (HANNON, 2003; GASSMAN; BECKER, 2006; SUN; LEUNG, 2007; SOUZA; SOUSA; BONILHA, 2008; RAUPP; 
BEREN, 2009). A partir dessa problemática, esta pesquisa busca responder à seguinte questão de pesquisa: como as Incubadoras de Base Tecnológica (IBT) contribuem para o desenvolvimento das capacidades e recursos das empresas incubadas em processo de incubação? Com o pressuposto de que este o processo de incubação será de extrema valia para o êxito e crescimento no mercado e com o objetivo de colaborar para aprofundar o conhecimento relacionado ao processo de incubação das empresas, este estudo tem como objetivo central analisar como as incubadoras de base tecnológica contribuem para o desenvolvimento das empresas incubadas nos diferentes estágios de incubação.

\section{Fundamentação teórica}

De acordo com a pergunta de pesquisa, foi realizada uma revisão teórica sobre RBV e sobre as incubadoras de negócios. Na seção que trata sobre a RBV, o foco são os recursos, as capacidades e como se desenvolvem nas empresas. A revisão sobre as incubadoras de empresas teve o foco nas de base tecnológica e no processo de incubação.

\subsection{Visão Baseada em Recursos (RBV)}

O termo resource-based view, ou visão baseada em recursos, ou ainda teoria dos recursos, foi utilizado pela primeira vez por Wernerfelt (1984), com a defesa do argumento que é mais proveitoso analisar empresas pelos recursos do que pelos produtos. Além disso, o autor foi o primeiro a defender uma visão organizacional fundamentada em recursos no campo da administração estratégica. A RBV consiste em uma abordagem de categorização e parte do pressuposto de que a empresa é proprietária de recursos que, combinados de forma exclusiva, podem gerar vantagem competitiva. A justificativa para realizar análises utilizando a Teoria dos Recursos baseia-se na tentativa de explicar a complexidade entre as fontes que geram em cada empresa as suas vantagens competitivas. 
Nos anos de 1990 a RBV conquistou destaque com a publicação de Barney (1991), em que o autor examinou a lacuna entre recursos empresariais e vantagem competitiva sustentável. Seu estudo teve como base duas suposições: heterogeneidade de recursos e imobilidade de recursos. Para Barney (1991), recursos são todos os ativos, capacidades, processos organizacionais, atributos empresariais, informação, conhecimento etc., controlados por uma empresa e que a habilitam a conceber e implementar estratégias que aprimorem sua eficiência e efetividade.

Ainda, verifica-se que seus estudos destacaram-se pelo modelo de análise de empresas: o VRIO (Valor, Raridade, Imitabilidade e Organização), que consiste em colocar os recursos internos da organização como potencializadores de vantagem competitiva sustentável, em vez de o ambiente externo, ou seja, analisa a empresa por suas capacidades internas para reconhecer, identificar e analisar os recursos geradores de vantagem competitiva sustentável. Para tanto os autores partem de quatro atributos essenciais: (V) ser valioso para que a empresa possa explorar uma oportunidade ou uma ameaça ambiental; (R) ser raro entre os concorrentes da empresa; (I) ser de difícil imitação pelos concorrentes; e (O) a empresa deve estar organizada para aproveitar ao máximo, o potencial desse recurso (BARNEY; HESTERLY, 2007).

\subsubsection{Capacidades}

Dentro da RBV, foi desenvolvida vertente que analisa as capacidades de uma empresa, em especial sua origem, formação e consolidação, pois há diferentes naturezas de capacidade, definidas como dinâmicas ou substanciais (ZAHRA; SAPIENZA; DAVIDSSON, 2006), mas alguns autores as classificam como nível zero (zero level) e nível primário (first order), mas com as mesmas definições correlatas (WINTER, 2003). E, ainda, o conceito de capacidades (principalmente as dinâmicas), complexo e que assume diversos papeis na administração estratégica. 
Os antecedentes das capacidades dinâmicas estão ligados ao fato de que gestores não criam soluções ou rotinas "únicas e para sempre", embora continuamente as revisem. De acordo com Zahra et al. (2006), há dois tipos de capacidades: as dinâmicas e as substanciais. Esses conceitos apresentam três elementos que se confundem na literatura: a habilidade de solucionar um problema, a presença de problemas que mudam rapidamente e a habilidade de alterar a maneira com que a empresa soluciona seus problemas.

O primeiro elemento é a própria definição de Capacidade Substancial: conjunto de habilidades e recursos utilizados para solucionar problemas ou conseguir um resultado. Para explicar capacidade substancial ou ordinária, deve-se fazê-lo em conjunto com as capacidades dinâmicas, definidas como habilidades de reconfiguração dos recursos e rotinas de uma empresa, com visão e julgada apropriada por seu tomador de decisão principal. Ou seja, para autores como Zahra et al. (2006), uma capacidade dinâmica é aquela que gera habilidade para modificar ou reconfigurar capacidades substanciais existentes. Uma nova rotina de geração de produtos é uma capacidade substancial, mas a habilidade de mudar essa rotina (capacidade substancial) é uma capacidade dinâmica. Para os autores, é difícil definir e separar esses dois conceitos sem causar redundância.

O segundo elemento se refere às características ambientais. Zahra et al. (2006) defenderam que um ambiente volátil ou mutável não é necessariamente componente de uma capacidade dinâmica. Os autores reconheceram que, em ambientes voláteis ou naqueles que sofreram mudanças drásticas, as empresas despendem de mais esforços para desenvolver e utilizar capacidades dinâmicas, transformando suas capacidades substanciais para atender às práticas mutáveis e instáveis do cenário competitivo em que atuam.

O terceiro elemento volta à discussão das definições de cada tipo de capacidade, porém examinando-as na relação com as demais. $\mathrm{Na}$ instância primordial das empresas, as capacidades substanciais precedem às dinâmicas. Com o tempo, a relação entre capacidades 
dinâmicas e substanciais torna-se complexa e coesa. Há uma crença de que essas capacidades ganham força com o uso e não se depreciam como ocorre com outros ativos da empresa.

Tal relação se desequilibra sempre que a empresa privilegia a utilização de um tipo de capacidade em detrimento de outro. Valerse continuamente das capacidades substanciais leva a empresa a despender mais esforço para desenvolver as capacidades dinâmicas que as modificarão. No entanto, a organização ganha dinamismo ao utilizar, de forma abusiva, de capacidades dinâmicas para aprimorar as substanciais, o que, em contrapartida, exigirá esforços redobrados para desenvolver novas capacidades.

Teece, Pisano e Shuen (1997), considerados legitimadores do conceito de "capacidades dinâmicas", defenderam que, para compreender adequadamente esse conceito, seria necessário analisar-lhe os elementos constitutivos: capacidades e dinâmica. 0 primeiro consiste em apropriadamente adaptar, integrar e reconfigurar habilidades, recursos e competências funcionais para adequar-se aos requerimentos de um ambiente mutável. Já o segundo se refere à capacidade de renovar competências para que se consiga congruência com o sempre mutável ambiente competitivo.

Convém ressaltar que, para Zahra et al. (2006), capacidades substanciais são mero conjunto de habilidades. Já para Teece et al. (1997), o simples termo é a habilidade de adaptar, integrar e reconfigurar as competências. Ou seja, há divergência no conceito para esses dois autores. Os primeiros autores afirmam que a capacidade é um simples termo nominativo que determina o tipo de habilidade, já os segundos autores, afirmam que as capacidades não nominam simplesmente, mas definem ação de adaptar, integrar e reconfigurar as habilidades (ou competências). No entanto, ao explicarem a terminologia dinâmica, os autores, ao contrário do que ocorreu no primeiro termo, convergem em uma mesma ideia: reconfiguração ou renovação de capacidades (competências e outros recursos) para responder a uma demanda de mudança. 
Nelson e Winter (1982), influenciados pelo pensamento de Schumpeter, abordaram a dicotomia de capacidades, afirmando que capacidade é uma rotina de alto nível (ou uma coleção de rotinas) que, com suas execuções de fluxos de entrada, produz para a gestão da empresa um conjunto de decisões que geram fluxos de saída particulares. Para explicar melhor esse conceito, argumentam que rotina provém de um aprendizado altamente padronizável, repetitivo e baseado em conhecimento tácito e na especificidade dos objetivos. Aqui, é possível ver que a capacidade é definida como algo quantificável, que possui fluxos de entrada e saída, porém sua definição ainda se baseia no conceito de uma habilidade aprendida e empregada. A grande contribuição do estudo é sua análise sobre como a mudança ocorre na organização. Os autores trataram dessa mudança, reduzindo-a a duas categorias (ou conjuntos de categorias): solução ad hoc de problemas e capacidades dinâmicas. Uma organização pode mudar utilizando soluções ad hoc de problemas como forma de "apagar o fogo". Esse tipo de mudança não é uma rotina, pois não há comportamento repetitivo e padronizado (WINTER, 2003). Outra maneira de a organização se transformar é por meio da capacidade dinâmica, considerada rotina, pois possui comportamentos necessários para ser classificada como tal.

As capacidades dinâmicas tiveram um enfoque maior no estudo de Peteraf e Helfat (2003). Nesse estudo, os autores afirmam que só será possível compreender, de fato, a vantagem competitiva se o conceito de evolução dos elementos que lhe dão sustentação - recursos e capacidades - for incorporado pela RBV. Para tanto, introduz-se o conceito de ciclo de vida das capacidades (capability life cycle), cuja importância é ajudar a explicar as fontes fundamentais de heterogeneidade (de recursos) de empresas. Os autores definem um padrão geral para esse ciclo, com base nas seguintes etapas: fundação, desenvolvimento e maturidade.

Barreto (2010), com foco nas capacidades dinâmicas, analisou o conceito de capacidades dinâmicas proposto por Teece et al. (1997), decompondo-o em cinco partes. Primeira, a natureza do conceito é categorizada como uma habilidade (ou capacidade). Segunda, 
especificou-se um fim para essa capacidade: integrar (ou coordenar), construir e reconfigurar competências externas e internas; portanto, os autores fundamentaram seus trabalhos de pesquisa na economia evolucionária. Terceira, houve foco em um contexto externo particular de mutação rápida, o que determinou o surgimento de uma perspectiva empreendedora. Quarta, as capacidades dinâmicas são, geralmente, construídas na organização e não meramente adquiridas. Quinta, as capacidades dinâmicas pressupõem heterogeneidade de recursos da RBV. Ressalte-se que nem todas as empresas da mesma indústria as possuem.

As capacidades componentes são extremamente importantes para a organização, pois delas dependem a criação e o aprimoramento da inimitabilidade de recursos (DIERICKX; COOL, 1989). Embora esse tipo de capacidade possa aumentar a eficiência organizacional, renderá à empresa inflexibilidade ante a inovação. Esforços de P\&D terão impacto negativo sobre a vantagem competitiva se as capacidades componentes não evoluírem de acordo com as mudanças ambientais.

\subsection{Incubadoras de empresas}

Incubadoras de empresas não são fenômenos recentes. A primeira ideia sobre o tema surgiu na Universidade de Stanford, em 1938, sendo, posteriormente, adotada pela Europa. No Brasil, em meados da década de 1970, na cidade de Campinas, a Universidade Estadual de Campinas (UNICAMP) começou o primeiro experimento, nos moldes dos parques tecnológicos americanos, com a criação da Companhia de Desenvolvimento Tecnológico - CODETEC (BIAGIO, 2006). No Brasil, a incubação de empresas, deixou de ser um fenômeno isolado, materializando-se em 377 incubadoras, em todas as regiões do país (PANORAMA ANPROTEC, 2006).

As experiências americanas, exemplificadas pelo Vale do Silício (Silicon Valley) e a Route 128, de acordo com Santos (1985), demonstram que a proliferação dessas indústrias de tecnologia avançada, fenômeno conhecido pelos americanos como high tech boom, 
permitiu o fortalecimento da competitividade de setores da economia nos mercados internacionais. Na Europa, tem experiências significativas na França (cité-scientifique), na Inglaterra (Sciences Parks) e na Alemanha (Berliner Innovations und Grunderzentrum).

A organização que possui o maior número de dados de Incubadoras de Negócios do Brasil é a ANPROTEC (Associação Nacional de Entidades Promotoras de Empreendimentos Inovadores), que em seus panoramas anuais traz dados relevantes. Como referência tem o último relatório com nível de detalhamento satisfatório (Figura 1).

Figura 1 - Número de incubadoras no Brasil - Evolução por região e postos de trabalhos gerados (2005)
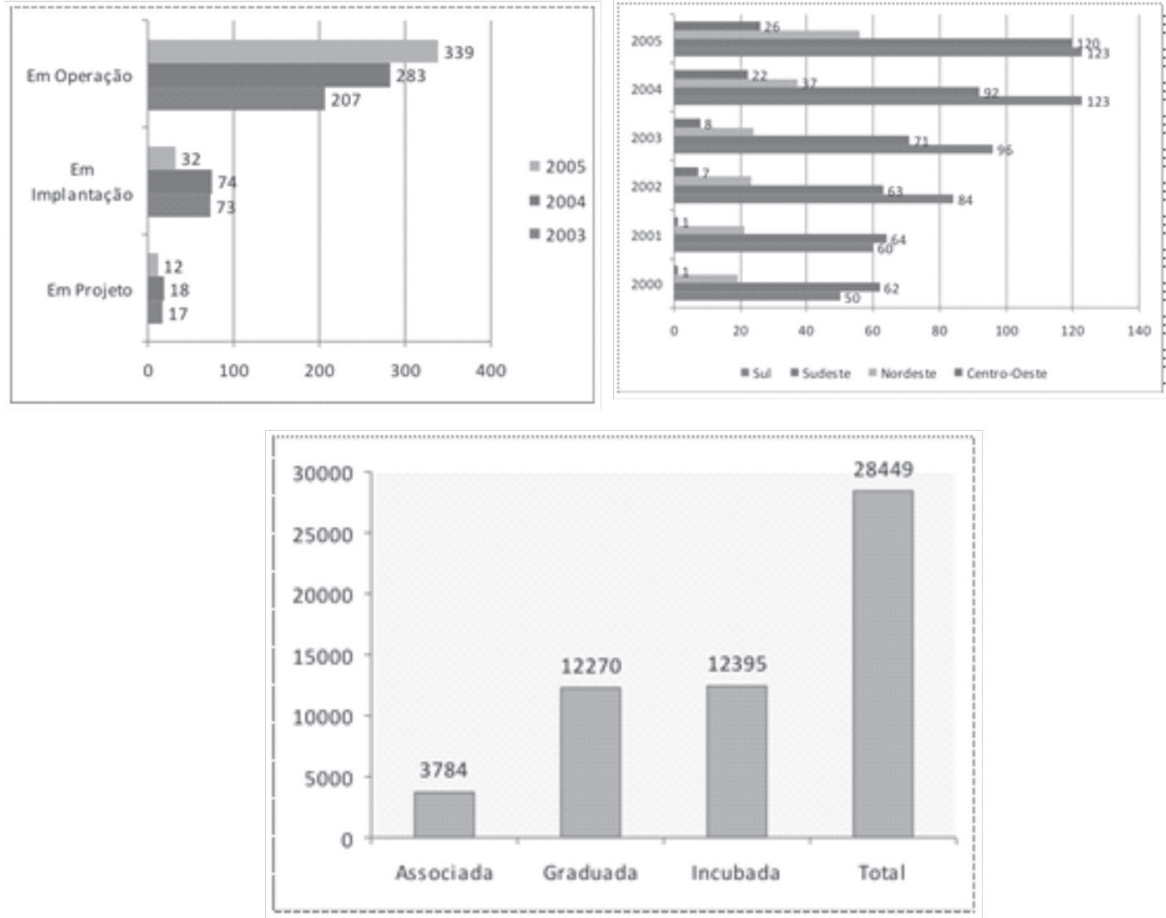

Fonte: ANPROTEC (2006).

Verifica-se, pelos dados, um grande aumento no número de incubadoras no Brasil, sob três aspectos: em operação, em implantação 
e em projeto. Ao longo dos anos, repara-se uma aglutinação natural de incubadoras em operação e um número decrescente de incubadoras em implantação e em projeto. Também é possível observar que a maioria das incubadoras se encontrava nas regiões Sul e Sudeste, porém, as regiões Nordeste e Norte apontaram massivo crescimento se comparado com as outras regiões. O número de postos de trabalho gerados pela incubadora é significativo e são distribuídos, da seguinte maneira: postos de trabalhos de empresas associadas a incubadoras, postos de trabalhos de empresas graduadas de incubadoras e postos de trabalhos de empresas incubadas de incubadoras. Destaca-se o equilíbrio dos postos de trabalho gerados por empresas graduadas e incubadas.

Para Lahorgue (2004), incubadoras podem ser classificadas em: a) incubadora de empresa de base tecnológica: organização que abriga empresas cujos produtos, processos ou serviços resultam de pesquisa científica, para a qual a tecnologia representa alto valor agregado; b) incubadora de empresas de setores tradicionais: organização que abriga empreendimentos ligados aos setores da economia que detêm tecnologias largamente difundidas e que queiram agregar valor aos seus produtos, processos ou serviços, por meio do incremento em seu nível tecnológico; e c) incubadora mista: organização que abriga ao mesmo tempo empresas de base tecnológica e de setores tradicionais.

Para Hannon (2003); Gassman e Becker (2006); Sun e Leung (2007); Souza, Sousa e Bonilha (2008); Raupp e Beren (2009), pelo processo de incubação, as capacidades e recursos são desenvolvidos na incubadora. Nesse processo, a empresa incubada recebe suporte e apoio para maximizar as possibilidades de sucesso do negócio. Há também o planejamento da inserção da incubada no mercado.

Hannon (2003) baseia-se na necessidade de desenvolver a incubadora em três grandes frentes: Ambiente de Incubação, Prospecção de Clientes eProcesso Empreendedor. Aprimeira,Ambiente de Incubação, refere-se a todos os recursos e infraestrutura disponíveis para as empresas incubadas durante seu período de incubação e fornecidos pela incubadora. A segunda, prospecção de clientes, se enquadra na atividade 
de suporte da incubadora, que auxilia nos aspectos mercadológicos e de produto a serem desenvolvidos na empresa incubada. A última frente, Processo Empreendedor, são atividades que a incubadora realiza para promover uma cultura e capacitação empreendedora em suas empresas incubadas. São palestras, seminários, workshops, capacitações etc. As frentes de desenvolvimento de uma incubadora geram impactos criação de capacidades - em empresas incubadas. Entre as capacidades desenvolvidas estão as seguintes: financeira, analítica, funcional do negócio, interpessoal e empreendedora.

Figura 2 - Etapas do processo de incubação

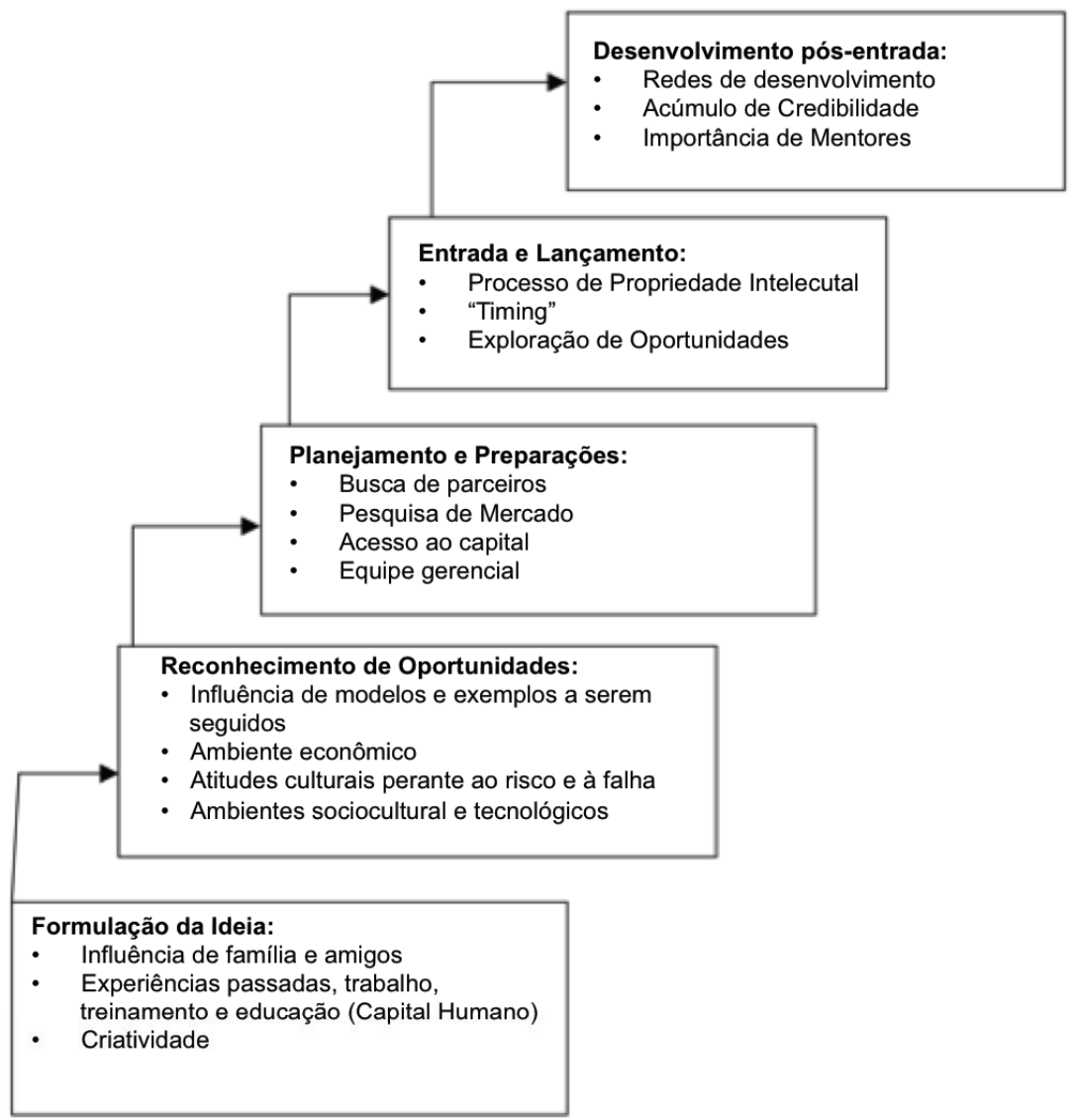

Fonte: Adaptado de Hannon (2003). 
Durante o processo de incubação (Figura 2), as empresas incubadoras formam certas capacidades (HANNON, 2003) em empresas incubadas por meio da oferta de recursos e serviços (SUN; LEUNG, 2007; SOUZA; SOUSA; BONILHA, 2008; RAUPP; BEREN, 2009; XU, 2010). Para Xu (2010), em seu estudo sobre incubadoras de base tecnológica na região de Shangai na China, estes recursos e serviços podem ser divididos em oito categorias de variáveis: Recursos Físicos ou Serviços relacionados à infraestrutura, Serviços de aprimoramento de credibilidade e visibilidade, Interação com a Gestão da Incubadora, Interação com Incubados, Acessibilidade e networking com recursos externos à incubadora, Serviços de Treinamento e Capacitação, Assistência específica de Negócio e Assistência para obtenção de financiamento externo. O estudo de Souza, Sousa e Bonilha (2008) elenca as seguintes categorias: Espaço Físico, Estratégia, ContábilFinanceira, Tecnologia de Informação, Operações e Recursos Humanos. Em um estudo mais amplo, Sun e Leung (2007) analisaram os fatores críticos de sucesso de incubadoras de base tecnológicas em Hong Kong, dividindo-os em três grandes categorias: relacionados ao ambiente, relacionados à incubadora e relacionados ao incubado.

Raupp e Beren (2009) abordam a questão dos serviços e recursos ofertados pela incubadora a seus incubados sob o ponto de vista das dificuldades e facilidades das empresas incubadas nas diversas fases do processo de incubação. As facilidades descritas pelos autores, em ordem de frequência, são: infraestrutura da incubadora, divulgação do produto, apoio da universidade, participação em eventos, redução de custos, oportunidades de negócios, acesso a pesquisadores, troca de experiências com outras empresas e consultorias. Já as dificuldades enfrentadas pelas empresas do estudo, seguem esta ordem: falta de recursos financeiros, falta de conhecimento em gestão empresarial, comercialização do produto, inserção da empresa no mercado, regularização da empresa, lançamento de novos produtos, gastos com impostos, fidelização de clientes e assessoria técnica.

Lee e Osteryoung (2004) desenvolveram um estudo semelhante ao de Xu (2010), com o foco nas incubadoras universitárias dos Estados 
Unidos e de Hong Kong, e descreveram 14 fatores que foram agrupados em quatro categorias, que para os autores são fundamentais para uma efetiva operação do processo de incubação:

- Estratégia e objetivos: (1) clareza no cumprimento dos objetivos; (2) estratégia de operação;

- Recursos físicos e humanos: (3) acesso fácil à infraestrutura e equipamentos; (4) acesso comunitário ao espaço de serviço e equipamentos de escritório; (5) network de suporte empreendedor; (6) experiência organizacional;

- Serviços da incubadora: (7) transferência de tecnologia e pesquisa e desenvolvimento ( $p \& d)$; (8) consultoria de negócios e legal; (9) suporte e consultoria financeira; (10) programa de educação empreendedora; e

- Programa de network: (11) network institucional; (12) network de incubados; (13) network de financiamento; (14) suporte local e do governo.

\section{Metodologia da pesquisa}

Segundo Martins e Theóphilo (2007, p. 37), "o objetivo da metodologia é o aperfeiçoamento dos procedimentos e critérios utilizados na pesquisa. Por sua vez, método é o caminho para se chegar a determinado fim ou objetivo". Dessa forma, considera-se que a metodologia da pesquisa é equiparada a uma preocupação instrumental em que a ciência busca captar a realidade, sendo que a metodologia trata de como isso pode der alcançado (Demo, 1995).

A metodologia nesta pesquisa baseia-se em estudos de casos múltiplos e de caráter exploratório conforme as recomendações de Eisenhardt (1989); Miles e Huberman (1994); Yin (2005) e Lima (2010). A proposta de Eisenhardt (1989) de elaborar estudo de casos em que os dados levam a conclusões indutivas é uma das inspirações metodológicas deste trabalho. Segundo a autora, a flexibilidade teórica deve ser um dos principais aspectos desta abordagem de análise, onde a pergunta de pesquisa e os constructos formulados antes da fase de 
coleta e entrada no campo de pesquisa devem ser maleáveis para que ideias que emergem da natureza empírica dos dados possam aprimorálos.

A relação entre incubados e incubadora, constructo com raízes na RBV, foi interpretada em dois eixos, sendo: a) os serviços prestados pela incubadora e b) pelo fluxo de recursos entre incubados e incubadora. Na pesquisa, a RBV foi explorada tendo como base os estudos de Lee e Osteryoung (2004); Sousa, Souza e Bonilha (2008); Raupp e Beren (2009); Xu (2010). Já os serviços que foram oferecidos durante o processo de incubação, tiveram como sustentação, para o presente estudo, as pesquisas de Hannon (2003). Já a relação pelo processo de incubação é fundamentada por Gassmann e Becker (2006).

Foram utilizados dois roteiros de entrevista para coleta de dados, sendo o primeiro, direcionado para incubadas e o segundo para o gestor da incubadora. Os casos foram analisados com base na contribuição teórica de cada caso para a saturação teórica do estudo. Foram escolhidas empresas em quatro estágios distintos de incubação no Centro de Inovação, Empreendedorismo e Tecnologia (CIETEC): pré-incubação, incubação inicial, incubação final e pós-incubação. A pós-incubação é uma modalidade seleta do CIETEC, já que não é contemplada em nenhum documento oficial divulgado. Essa modalidade de incubação é composta por empresas que foram incubadas, porém, desejam manter o vínculo após a graduação com o CIETEC e objetivam manter-se dentro do ambiente CIETEC-USP. Foi também tomado o cuidado de serem escolhidas empresas que produzem produtos de naturezas diferentes para eliminar quaisquer vieses não sistemáticos.

Para a coleta de dados foram realizadas entrevistas semiestruturadas, elaborados com base nos ensinamentos de Flick (2009), com os sócio-fundadores das empresas participantes da pesquisa para análise e com o gestor da incubadora. Assim, as entrevistas foram conduzidas de forma aberta, sem a necessidade de realizá-las de forma padronizada, o que permitiu maior descontração entre os entrevistados e os pesquisadores. 


\section{Análise e discussões dos resultados}

Conforme definido na metodologia, procedeu-se uma pesquisa com o gerente da Incubadora e com quatro empresas em diferentes estágios de incubação com o objetivo de responder à pergunta de pesquisa.

Para melhor organização desta seção, abordam-se as quatro dimensões da pesquisa de forma separada em: (1) apresentação da incubadora e dos casos; (2) análise da relação incubadora-incubado, com foco na teoria de capacidades e recursos (RBV); e (3) caracterização das contribuições das incubadoras nos diferentes estágios de desenvolvimento dos incubados.

\subsection{Apresentação da incubadora e dos casos}

Para este estudo, foi selecionada a incubadora: Centro de Inovação, Empreendedorismo e Tecnologia (CIETEC), localizado no município de São Paulo; e quatro empresas: Home Solutions; NPT NeuroPsicotronics; CHEM4U; e Brasil Ozônio; que se encontravam em diferentes estágios de incubação.

\subsubsection{Incubadora CIETEC}

A incubadora escolhida para análise das empresas e empresários incubados foi o CIETEC que possui uma diversidade de empresas incubadas. A escolha do CIETEC foi motivada pela facilidade de se encontrar empresas de vários setores em diferentes estágios de incubação. Criado em 1998 e com sede no campus do IPEN - Instituto de Pesquisas Energéticas e Nucleares, no coração da Cidade Universitária - da Universidade de São Paulo (USP), o CIETEC tem como missão incentivar o empreendedorismo e a inovação tecnológica e apoiar a criação, fortalecimento e consolidação de empresas e empreendimentos inovadores, de base tecnológica. A incubadora contempla quatro diferentes categorias de associação: (1) hotel de projetos; (2) Residente 
Tecnológico (RT); (3) Residente de Tecnologia da Informação e Comunicação (TIC); e (4) não residentes.

As três primeiras categorias são empresas que estão estabelecidas na incubadora e a quarta categoria, é composta por empresas que possuem sua sede de operação fora do CIETEC, porém desfrutam dos serviços oferecidos. De acordo com dados coletados em abril de 2012, o CIETEC possuía um total de 125 empresas associadas. A seleção das empresas e empresários para associação se dá por intermédio de um processo de seleção bem estruturado, definido, institucionalizado e composto por um fluxo de etapas de aprovação em que a proposta é analisada até sua aprovação final.

\subsubsection{Empresas incubadas}

Home Solutions: associada na modalidade de hotel de projetos (pré-incubação), ingressou no CIETEC em março de 2012. Tem como produto um serviço denominado de "Gestão de Vidas", uma espécie de governança da vida de pessoas. A filosofia do serviço se baseia realizar tarefas simples como compras supermercados, administração doméstica entre outras, pois em um mundo cada vez mais veloz em que as pessoas estão cada vez mais atarefadas e ocupadas com atividades profissionais e diversas, elas não conseguem dar atenção a estas tarefas consideradas "simples", mas que demandam tempo e atenção das pessoas. Adicionalmente, o serviço também engloba como clientes potenciais pessoas que possuem problemas com a organização de seus afazeres e pessoas idosas que não conseguem realizar estas tarefas sozinhas.

NPT - NeuroPsicotronics: foi incubada em agosto de 2010 e encontra-se em fase inicial de incubação na modalidade "residente TIC". A empresa desenvolve um produto denominado CardioEmotion, que tem por finalidade conscientizar e monitorar pessoas sobre o seu nível de stress. O software também tem a finalidade de capacitar uma pessoa à controlar seus batimentos cardíacos 
CHEM4U: desenvolvedora de vernizes com nanotecnologia na indústria química e se encontra em estágio final de incubação, a empresa iniciou sua incubação em 2007 e conta com uma infraestrutura própria. O uso de nanotecnologia é o grande diferencial da empresa no setor em que compete.

Brasil Ozônio: A empresa começou sua incubação em 2004 e, depois que se graduou, decidiu permanecer na incubadora como pós-incubado. O negócio da organização é a sanitização com o uso do ozônio e atua em diversas frentes como: alimentos, ambientes hoteleiros, ambientes hospitalares, tratamento de água, piscinas e poços artesianos. A atratividade do serviço se dá pelo impacto ambiental que gera, pois o resíduo resultante do processo de sanitização por ozônio são água e oxigênio.

\subsection{Relação incubadora-incubado}

O começo da relação entre incubadora e incubados se dá no processo de seleção. Os empreendedores destacaram que durante o processo de incubação não houve aplicação de entrevista pela incubadora. Este fato foi considerado negativo pelos incubados, pois os incubados destacam que a entrevista pelo CIETEC é necessária quando não há algo muito bem explicado no plano de negócios, geralmente no que tange a assuntos técnicos do produto.

Assim, os empreendedores que são selecionados para a próxima etapa, o workshop de plano de negócios, com a exceção do empreendedor da Brasil Ozônio, relataram que esta etapa foi essencial para a aprovação da proposta pela incubadora. Os entrevistados enalteceram a capacidade do workshop em expandir seus horizontes de planejamento e visão do negócio, estruturando-os em um plano de negócios objetivo, coerente e com um cronograma de ações que servirão de guia para quando lançarem suas atividades empresariais. Inclusive, os incubados são avaliados periodicamente e, caso não estejam de acordo com os seus planos de negócios, podem ser retirados do processo de incubação. De acordo com Hannon (2003), em seu estudo 
em que descreve as cinco etapas do processo de incubação, o processo de seleção do CIETEC abrange três dessas cinco etapas: formulação da ideia; reconhecimento de oportunidades; e planejamento e preparações. Dentro dessas, a etapa do workshop de plano de negócios é a grande "espinha dorsal" que estrutura o negócio.

Os empreendedores iniciantes, em pré-incubação e início da incubação, responderam que o processo de incubação não teve grandes impactos que pudessem ocasionar uma mudança no que já estava previsto em seus planos de negócios. Já os empreendedores em fase final de incubação ou em pós-incubação atribuem uma forte alteração em seus produtos e em suas empresas durante o processo de incubação. Essas mudanças podem ser analisadas como consequências diretas da entrada gradual no mercado, ou seja, o que ocasionou as mudanças foi o contato inicial da empresa e de seus produtos com o mercado de maneira mais ampla (concorrentes, fornecedores, clientes, credores etc.); e não o processo de incubação em si (AUTIO; GEORGE; ALEXY, 2011).

Esses impactos geraram mudanças nas empresas com maior tempo de incubação e ainda não foram sentidas na sua plenitude nas empresas com menor tempo de incubação podendo serem correlacionadas com a quarta etapa do processo de incubação descrita por Hannon (2003): entrada e lançamento; momento em que as oportunidades são exploradas. Como empresas com maior tempo de incubação tendem a ter um grande número de oportunidades já exploradas do que empresas com menor tempo de incubação, estas empresas estão sujeitas a maiores mudanças devido à amplitude de cobertura do mercado e maior tempo de contato com os clientes.

Os empreendedores que estão em processo inicial de incubação identificam a infraestrutura como algo essencial, pois ajudam a dar foco ao que interessa para eles: o produto e o negócio. Já os empreendedores estabelecidos, em fase final de incubação e em pós-incubação, enxergam a infraestrutura como algo que não pode ser relacionada ao sucesso da empresa. A empreendedora da CHEM4U, em fase de incubação final, afirmou que a principal vantagem da infraestrutura oferecida pela 
incubadora é justamente o ambiente de troca de experiências que o CIETEC proporciona, o que é chamado por Lee e Osteryoung (2004) de network off-line. Neste aspecto foi possível constatar um choque de realidades que os casos analisados do CIETEC em relação aos achados na literatura levantada, como, por exemplo, nos estudos de Lee e Osteryoung (2004); Souza, Sousa \& Bonilha (2008); Raupp e Beren (2009); Xu (2010). Nesse sentido, foi possível observar a grande importância que foi atribuída à infraestrutura como um dos serviços primordiais que as incubadoras oferecem para o estabelecimento do negócio das empresas incubadas. Porém, as respostas de dois (fase final e pós-incubação) dos quatro empreendedores incubados contrastavam; e concluíram que a infraestrutura é apenas "a cortina do espetáculo". Essa constatação evidencia que quanto mais inicial está o estágio de incubação, mais as empresas incubadas necessitam da infraestrutura. O que não ocorre nos estágios finais, em que o network proporcionado pela incubadora e as mudanças no mercado de atuação da empresa passam a ser importantes.

Quanto aos serviços da incubadora que os respondentes consideraram primordiais para o estabelecimento do negócio, foram observadas respostas, que apesar de bem diversificadas apresentaram um padrão que é justamente a possibilidade de se estabelecer o networking. Cada resposta tem um lastro com a natureza do negócio e da empresa em questão. A HomeSolutions utilizou apenas poucos serviços da incubadora, pois na época da coleta, estava na há apenas há dois meses no CIETEC. O NPT - NeuroPsicotronics, como é uma empresa focada em vendas, precisava de uma maneira sustentada e ordenada para expandir a sua rede de relações. Já o CHEM4U, como foi constituído por dois químicos de formação com experiência na área de pesquisa e desenvolvimento (P\&D), necessitava ter acesso a capacitações empresariais como forma de complementar as formações dos sócio-fundadores. Já a Brasil Ozônio, como possuía redes de relacionamento com organizações de apoio, como escritório de contabilidade, escritórios de advocacia entre outros; e devido ao ineditismo do seu negócio, o ozônio, necessitava, de acordo com Xu (2010), de serviços de aprimoramento de credibilidade e visibilidade. 
Com a análise das respostas à luz da teoria destaca-se a maturidade da literatura coletada, pois nenhum serviço aqui destacado é inédito para o referencial teórico, pois foram previstos em estudos recentes (LEE; OSTERYOUNG, 2004; SOUZA; SOUSA; BONILHA, 2008; RAUPP; BEREN, 2009; XU, 2010). Houve o destaque, para a questão do network off-line de incubados; que foi respondido por dois empreendedores que se encontravam tanto em estágio inicial quanto final de incubação.

Com o embasamento no estudo de Gassmann e Becker (2006), que aplica a teoria RBV ao analisar o fluxo de recursos de incubadoras corporativas, foram questionados aos entrevistados os retornos que as empresas geraram para a Incubadora e o funcionamento dessa parceria e para os entrevistados o fluxo privilegia a "empresa-mãe", ou seja, a corporação que sustenta a incubadora e seus incubados, pois é essa organização que define o escopo temático das propostas de incubação de acordo com os temas prioritários de serem desenvolvidos dentro da empresa.

A última pergunta do roteiro de entrevista serviu como uma pergunta-controle, em que foi possível verificar a existência ou não de uma vantagem plena para empresas que são incubadas na incubadora estudada e não verificar a qualidade da vantagem já presumida. Todos os entrevistados responderam que não conseguem imaginar o negócio sem o apoio da incubadora. Cabe destacar que para as empresas em fase final de incubação e pós-incubação deram pouca importância à infraestrutura ofertada pelo CIETEC, mas afirmaram que o networking proporcionado e o apoio recebido pelo CIETEC são fundamentais para o desenvolvimento do negócio.

\subsection{Contribuições das incubadoras no desenvolvimento dos incubados}

Na relação incubadora-incubado, contemplada à luz teórica da RBV, houve ilações significativas. A primeira de que o processo de seleção, mais especificamente no workshop de plano de negócios, foi 
essencial para o estabelecimento do negócio e direcionou a capacidade de planejamento e execução das empresas incubadas. Na percepção dos incubados, eles que detinham o controle das decisões e atividades da empresa, demonstrando que o papel desempenhado pela incubadora era mais passivo do que era esperado e que a incubação era reduzida a apenas um sistema coletivo de competências e recursos. Houve, também, divergência dos resultados obtidos a respeito da percepção da infraestrutura oferecida pela incubadora com relação ao que é retratado pela literatura do assunto. Descobriram-se fortes evidências entre os incubados, pois os que estavam em fase inicial de incubação davam mais importância em relação à infraestrutura física. Já os que estavam em fases mais avançadas do processo de incubação, percebiam pouca ou nenhuma importância da infraestrutura oferecida pelo CIETEC. Porém, todos os empreendedores foram unânimes em relação à importância do networking proporcionado pela incubadora. Esses achados vêm ao encontro do que se discute na literatura sobre RBV, e a diferença entre capacidades em empresas já estabelecidas e em empresas jovens (AUTIO COLLIS, 1994; ZAHRA et al., 2006; GEORGE; ALEXY, 2011).

A respeito de quais serviços ofertados durante o processo de incubação foram primordiais no estabelecimento do negócio também houve divergência nas respostas dos entrevistados, em que cada um foi tendenciado de acordo com a natureza e foco da gestão empresarial da empresa e, principalmente, de cada estágio do processo de incubação.

\section{Conclusão}

Dentro da teoria dos recursos, RBV, há um campo que estuda as capacidades de empresas. Nesse campo existe uma corrente influenciada pela escola da economia evolucionária, que define capacidades como uma rotina de alto nível (ou uma coleção de rotinas) que, com suas execuções de fluxos de entrada, produzem para a gestão da empresa um conjunto de decisões, gerando fluxos de saída particular (NELSON; WINTER, 1982; WINTER, 2003). 
Com o objetivo de responder à questão de pesquisa: como as Incubadoras de Base Tecnológica (IBT) contribuem para o desenvolvimento das capacidades e recursos das empresas incubadas em processo de incubação? Foi realizada a análise sobre a relação entre incubadora-incubada com o foco na teoria de capacidades e recursos (RBV), verificando-se que a capacitação dos incubados durante o processo de seleção, com o intuito de aperfeiçoamento do desenvolvimento de plano de negócios, é percebida como fator que contribuiu positivamente e primordialmente para o estabelecimento do negócio, pois direciona a capacidade de planejamento e execução das empresas incubadas.

Além disso, o processo de incubação demonstrou um papel mais passivo que o esperado, pois a percepção dos incubados é de que eles eram os que detinham o controle de todas as decisões e atividades da empresa e que o processo de incubação era apenas um sistema coletivo de recursos e competências que somente deve ser acionado durante momentos que nenhuma alternativa lógica se apresenta.

Enquanto que para as empresas nos estágios iniciais do processo de incubação a infraestrutura é percebida como elemento fundamental, nas empresas em estágios mais avançados de incubação, a infraestrutura é considerada com pouca importância. Porém, o networking proporcionado por ela é essencial para o desenvolvimento dos negócios.

Este estudo apresenta algumas limitações. Primeiro, os dados coletados são de apenas uma incubadora e de quatro empresas incubadas, portanto as análises dos fenômenos estudados não são passíveis de generalizações. Segundo, o município em que a incubadora e as empresas incubadas se situam, São Paulo, é muito diverso, contribuindo para o rol de fatores que podem influenciar o estudo. Por fim, o estudo é transversal e não possui dados com características longitudinais; o que enaltece vieses da pesquisa, principalmente na questão da formação de capacidades.

Nossas contribuições são para a ampliação dos contextos em que capacidades podem ser estudadas. O fenômeno de incubação 
de empresas é pouco pesquisado à luz de teorias consolidadas como a RBV, entretanto, vê-se grande contribuição deste fenômeno para o enriquecimento teórico, pois incubadoras e suas empresas incubadas propiciam um ambiente favorável à pesquisa, no qual os estímulos da incubadora às suas empresas incubadas são facilmente identificados e analisados.

Como sugestão de estudos futuros, identifica-se a falta de pesquisas sobre o processo de incubação de empresas em incubadoras brasileiras, também a grande escassez sobre como incubadoras selecionam suas empresas e seus empreendedores. O fenômeno necessita de mais estudos quantitativos ou longitudinais que possuam potencial de propor generalizações. Adicionalmente, percebem-se oportunidades de estudos que relacionem empresas incubadas ou incubadoras de diferentes países, com o propósito de revelar eventuais diferenças culturais e sociais que possam ter potenciais contribuições, tanto empíricas quanto teóricas.

\section{Referências}

BRASÍLIA (DF). ANPROTEC-Agência Nacional de Entidades Promotoras de Empreendimentos Inovadores. Panorama 2006. Disponível em <http://www.anprotec.org.br/publicacaopanorama. php?Idpublicacao=208>. Acesso em 15 nov. 2009.

AUTIO, E.; GEORGE, G.; ALEXY, O. International Entrepreneurship and Capability Development - Qualitative Evidence and Future Research Directions. Entrepreneurship Theory and Practice, Waco, Texas, v 35 , n.1, p. 11-37, jan. 2011.

BARRETO, I.. Dynamic Capabilities: A Review of Past Research and an Agenda for the Future. Journal of Management, Columbia, SC, v.36, n.1, p. 256-280, jan. 2010.

BARNEY, J. B. Firm Resources and Sustained Competitive Advantage. Journal of Management, Columbia, SC, v.17, n.1, p. 99-120, mar.1991. 
BARNEY, J B.; HESTERLY, W. S. Administração Estratégica e Vantagem Competitiva. São Paulo: Pearson Prentice Hall, 2007.

BAÊTA, Adelaide Maria Coelho; BORGES, Candido Vieira; TREMBLAY, Diane-Gabrielle. Empreendedorismo nas incubadoras: reflexões sobre tendências atuais. Comportamento Organizacional e Gestão, Lisboa, v.12, n.1, p. 7-18, 2006.

BIAGIO, L. A. Incubadoras de empreendimentos orientados para o desenvolvimento local e setorial: planejamento e gestão. Brasília: ANPROTEC - SEBRAE, 2006.

COLLIS, D. J. Research note: how valuable are organizational capabilities? Strategic Management Journal, Malden, MA, v. 15, Supplement S1, p. 143-152, Winter, 1994.

DEMO, P. Metodologia científica em ciências sociais. 3. ed. São Paulo: Atlas, 1995.

DIERICKX, I.; COOL, K. Asset Stock Accumulation and Sustainability of Competitive Advantage. Management Science, Catonsville, MD, v. 35, n.12, 1504-1511, Dec.1989.

FLICK, U. Introdução à pesquisa qualitativa. Porto Alegre: Artmed, 2009.

GASSMANN, O.; BECKER, B. Towards a resource-based view of corporate incubators. International Journal of Innovation Management, Singapore, v.10, n.1, p. 19-45, Mar. 2006.

HANNON, P. D.. A conceptual development framework for management and leadership learning in the UK incubator sector. Education + Training, Reino Unido, v. 45, n. 8/9, p. 449-460, 2003.

HELFAT, C. E.; PETERAF, M. A. The dynamic resource-based view: capability lifecycles. Strategic Management Journal, Malden, MA, v. 24, n.10, p. 997-1010, Oct. 2003. 
LAHORGUE, M. A. Parques, pólos e incubadoras: Instrumentos de desenvolvimento do século XXI. Brasília: ANPROTEC/SEBRAE, 2004.

LEE, S. S.; OSTERYOUNG, J. S. A comparison of Critical Success Factors for Effective Operations of University Business Incubators in the United States and Korea. Journal of Small Business Management, [S.I.], v.42, n.4, p. 418-426, Oct. 2004.

LIMA, E.. Teorizando a partir de dados qualitativos em administração. Pretexto, Belo Horizante, v.11, n.1, p. 73-93, jan./mar. 2010.

MCADAM, M.; MARLOW, S. . Building Futures or Stealing Secrets?: Entrepreneurial Cooperation and Conflict within Business Incubators. International Small Business Journal, s.I., v. 25, n.4, p. 361-382, Aug. 2007.

MARTINS, G. A.; THEÓPHILO, C. R.. Metodologia da Investigação Científica para Ciências Sociais Aplicadas. Atlas: São Paulo, 2007.

NELSON, R. R.; WINTER, G. S.. An evolutionary theory of economic change. Belknap press: Boston, 1982.

RAUPP, F. M.; BEUREN, I. M.. Programas oferecidos pelas incubadoras brasileiras às empresas incubadas. Revista de Administração e Inovação, São Paulo, v.6, n.1, p. 83-107, jan./abr., 2009.

SANTOS, S. A.. A criação de empresas industriais de tecnologia avançada: a experiência européia e as perspectivas brasileiras. Revista de Administração, São Paulo, v. 20, n.3, p.10-16, jul./set. 1985.

SERVIÇO BRASILEIRO DE APOIO ÀS MICRO E PEQUENAS EMPRESas. Taxas de Sobrevivência das Empresas no Brasil. Disponível em: <http://www.biblioteca.sebrae.com.br/bds/BDS.nsf/454 65B1C66A6772D832579300051816C/\$File/NT00046582.pdf>. Acesso em 15 Nov. 2009. 
SOUZA, J. H.; SOUSA, J. E. R.; BONILHA, I. D.. Avaliação do Processo de Incubação no Estado de São Paulo. Revista da Micro e Pequena Empresa, Campo Limpo Paulista, v. 2, n. 2, p. 21-39, maio/ago. 2008

SUN, H.; NI, W.; LEUNG, J.. Critical success factors for technological incubation: case study of Hong Kong Science and Technology Parks. International Journal of Management, [S.I.], v. 24, n.2, p. 346-363, jun. 2007.

TEECE, D. J.; PISANO, G.; SHUEN, A.. Dynamic capabilities and strategic management. Strategic Management Journal, Chicago, v. 18, n. 7, p. 509-533, Aug.1997.

TRIVIÑOS, A. N. S.. Introdução à pesquisa em ciências sociais: a pesquisa qualitativa em educação (o positivismo, a fenomenologia, e o marxismo). Atlas: São Paulo, 1987.

WERNERFELT, B..A Resourced-based view of the firm. Strategic Management Journal, Chicago, v. 5, n. 2, p. 171-180, abr./jun.1984.

winter, s. g.. understanding Dynamic Capabilities. Strategic Management Journal, Chicago, v. 24, n.10, p. 991-995, Oct. 2003.

XU, L.. Business Incubation in China: Effectiveness and perceived contributions to tenant enterprises. Management Research Review, Reino Unido, v. 33, n.1, p. 90-99, 2010.

YIN, R. K.. Estudo de Caso: planejamento e métodos. 3. ed.. Porto Alegre: Bookman, 2005.

ZAHRA, S.; SAPIENZA, H. J.; DAVIDSSON, P. (2006). Entrepreneurship and dynamic capabilities: a review, model and research agenda. Journal of Management Studies, Malden MA, v. 43, n. 4, p. 917-955, Jun. 2006.

Artigo recebido em: 11/02/2015

Aprovado em: 15/06/2015 